\title{
Microanalytical Standards, Reference and Research Materials: Continuing the Effort toward Breaking the Accuracy Barrier
}

\author{
Anette von der Handt ${ }^{1}$, Julien Allaz ${ }^{2}$, Owen K. Neill ${ }^{3}$ \\ ${ }^{1}$ University of Minnesota, Dept. of Earth Sciences, Minneapolis (MN), USA \\ ${ }^{2}$ University of Colorado Boulder, Dept. of Geological Sciences, Boulder (CO), USA \\ ${ }^{3}$ Peter Hooper GeoAnalytical Lab, Washington State University, Pullman (WA), USA
}

Reference materials and standards play a critical role in quantitative microbeam methods, as they are necessary for instrumental calibration, quality assurance (e.g. secondary standards) and inter-laboratory comparison. Certified materials ("good" standards) are available from a handful of providers, having been tested for micro-homogeneity and reference composition through independent methods and round robins. However, this process is time consuming and costly [1]. Hence, most reference materials in use are not formally certified, and many analysts use compilation values or their own preferred values.

This situation is complicated further, as compositional values of certified standard materials, tested by the best methods available at the time of their release, have been revised and updated [e.g. 2, 3] leading to labs potentially using a mix of old and updated values. New reference materials are also continuously being developed [e.g. 2, 4, 5] and existing materials are becoming more widely disseminated.

Over the last years, it has been shown that not all widely used materials fully comply with the rigorous criteria of "good" standards but rather fall into the categories of "bad" and "ugly" for various reasons [6]. In the case where subpopulations of a material exist that deviate from the published values, careful investigation and assessment for internal consistency among a suite of standards can identify such outliers. Modifying database compositions accordingly or excluding individual grains from analytical protocols can then improve on accuracy. In other cases, some standards can still provide accurate data with specific analytical protocols such as using defocused electron beams to minimize the effect of submicron inclusions [7], averaging over multiple grains to minimize intergrain heterogeneity [8], tracking time dependent intensity changes for beam sensitive standards [9], or accounting for peak shape changes between materials [10]. Good characterization of one's own standard collection, knowledge of compositional variation between nominally identical materials sourced from different providers [11], and adhering to proper standard maintenance protocols [6] is key to EPMA accuracy and direct comparison of analytical results from different laboratories.

Generating this level of documentation for a wide range of materials is often beyond the scope of what a single lab or standard provider can perform. Furthermore, the lengthy time scales for the certification process often lag behind the needs of the community. The amount of information on individual standards and reference materials has significantly increased in recent years. Unfortunately, such information tends to be either scattered over individual publications, conference abstracts and personal anecdotes or is inconsistently or, even worse, erroneously reported.

Moreover, recent hardware and software innovations will necessitate further characterization of reference materials. Hardware innovations include brighter electron sources (Schottky emitters), new WDS monochromator materials, modern SDD-EDS and a new generation of soft X-ray spectrometers (SXES, Multispectral glasses) facilitating low voltage analysis and the use of non-traditional X-ray 
lines. Software innovations are establishing new benchmarks in terms of improved precision, accuracy and productivity utilizing time-dependent intensity corrections, mean atomic number as well as multipoint background acquisitions and blank corrections. Accordingly, currently available and new standards and reference materials will have to be investigated for additional parameters such as nanoscale inclusions or inhomogeneities as well as oxidation and chemical bonding states.

Therefore there is a continued and increasing need for communication within the microbeam community in order to consolidate existing information about available standards and reference materials, and to curate analytical and maintenance protocols. For this purpose a new Focused Interest Group on MicroAnalytical Standards (FIGMAS [12]: https://figmas.org) was established under the umbrella of the Microanalysis Society and Microscopy Society of America. A key element is a database that gathers published and unpublished analytical data together with relevant metadata and sourcing options for available reference materials in a form that can be readily accessed by the analytical community. This can then provide a fresh avenue to disseminate information, establish guidelines for critical evaluation of one's own standards and help make informed decisions for future acquisitions of standard materials. Ideally this effort will be complimented with round robins to demonstrate instrumental performance, verify or establish the homogeneity and accuracy of new and existing reference materials, and further establish analytical protocols for reference material development and characterization.

\section{References}

[1] RB Marinenko, J. Res. Nat. Inst. Stand. Tech. 107 (2002), pp 687-691.

[2] J Donovan et al., Canadian Mineralogist 41 (2003), pp 221-232.

[3] B Wagner et al., Anal. bioanal. chemistry 402 (2012), pp 1667-1677.

[4] PK Carpenter et al., J. Res. Nat. Inst. Stand. Tech. 107 (2002), pp 703-718.

[5] EP Vicenzi et al., J. Res. Nat. Inst. Stand. Tech. 107 (2002), pp 719-727.

[6] PK Carpenter, Microsc. Microanal 14 (2008), pp 530-531.

[7] PK Carpenter, EP Vicenzi, Microsc. Microanal. 18 (2012), pp 1734-1735.

[8] J Fournelle, AGU Fall Meeting Abstracts (2012), V23C-2827.

[9] J Donovan, Topical conference on Microanalytical Reference Materials (2012).

[10] J Fournelle, CA Geiger. AGU Fall Meeting abstract (2010), V51C-2208.

[11] J. Fournelle, AGU Fall Meeting Abstracts (2009), V31E-2009.

[12] JM Allaz et al., Microsc. Microanal, 21 (2015), pp 2019-2020.

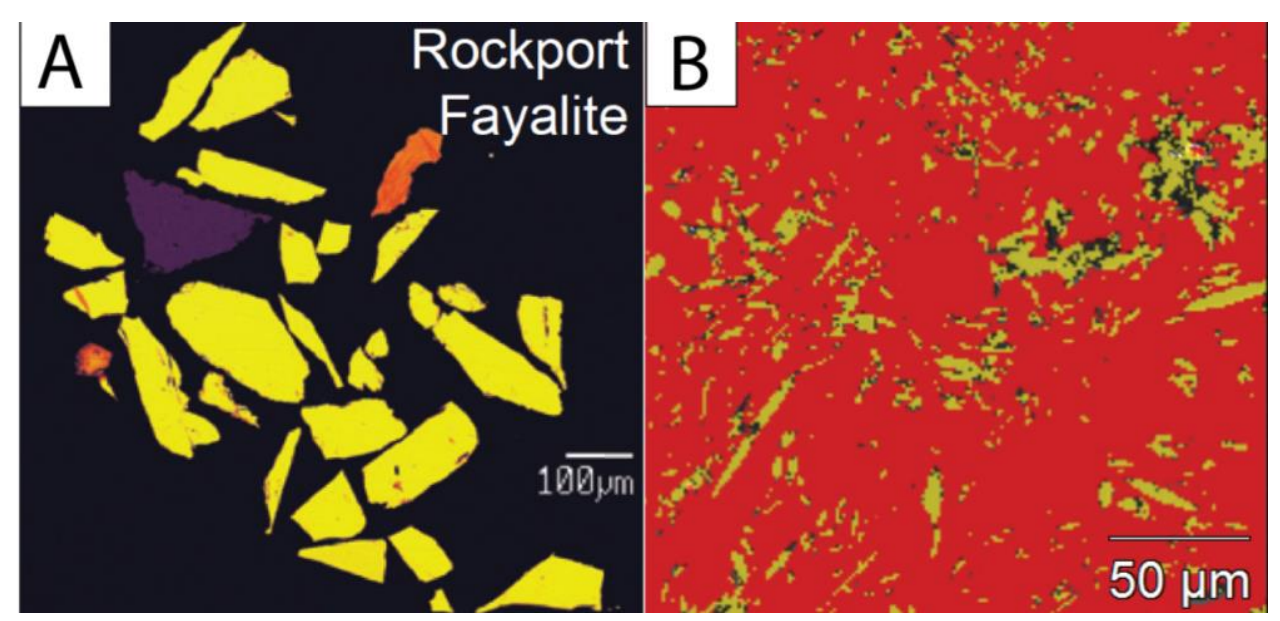

Figure 1. Examples of "bad" standards. A: NMNH 85276 (Rockport Fayalite) often containing a significant proportion of grunerite; B: Benitoite (red) (C.M. Taylor) containing abundant crossite (yellow) inclusions. 